\title{
Spectroscopy of planetary nebulae in M $33^{\star}$
}

\author{
L. Magrini ${ }^{1}$, M. Perinotto ${ }^{1}$, R. L. M. Corradi ${ }^{2}$, and A. Mampaso ${ }^{3}$ \\ 1 Dipartimento di Astronomia e Scienza dello Spazio, Universitá di Firenze, L.go E. Fermi 2, 50125 Firenze, Italy \\ 2 Isaac Newton Group of Telescopes, Apartado de Correos 321, 38700 Santa Cruz de La Palma, Canarias, Spain \\ 3 Instituto de Astrofísica de Canarias, c. Vía Láctea s/n, 38200, La Laguna, Tenerife, Canarias, Spain
}

Received 7 August 2002 / Accepted 29 November 2002

\begin{abstract}
Spectroscopic observations of 48 emission-line objects of M 33 have been obtained with the multi-object, wide field, fibre spectrograph AF2/WYFFOS at the $4.2 \mathrm{~m}$ WHT telescope (La Palma, Spain). Line intensities and logarithmic extinction, $c_{\beta}$, are presented for 42 objects. Their location in the Sabbadin \& D'Odorico diagnostic diagram (H $\alpha /[\mathrm{S}$ II] vs. H $\alpha /[\mathrm{N}$ II]) suggests that $>70 \%$ of the candidates are Planetary Nebulae (PNe). Chemical abundances and nebular physical parameters have been derived for the three of the six PNe where the $4363 \AA$ [O III] emission line was measurable. These are disc PNe, located within a galactocentric distance of $4.1 \mathrm{kpc}$, and, to date, they are the farthest PNe with a direct chemical abundance determination. No discrepancy in the helium, Oxygen and Argon abundances has been found in comparison with corresponding abundances of PNe in our Galaxy. Only a lower limit to the sulphur abundance has been obtained since we could not detect any [S III] line. N/H appears to be lower than the Galactic value; some possible explanations for this under-abundance are discussed.
\end{abstract}

Key words. planetary nebulae: individual: M 33 - galaxies: individual: M 33 - galaxies: abundances

\section{Introduction}

Chemistry of galaxies has been studied for a long time using spectroscopy of the integrated stellar light. There are, however, limitations to this method, such as the large abundance variations from star to star and the consequent difficulty in the interpretation of the data (McWilliam 1997), or the impossibility of using this technique in poorly populated regions, as in the haloes of elliptical galaxies.

Planetary nebulae $(\mathrm{PNe})$ with their prominent emission lines can be used to measure the metallicity in nearby galaxies. In spite of the importance of the topic, few spectroscopic data have been obtained for PNe in external galaxies, except for the Magellanic Clouds. PNe in the LMC and the SMC have been extensively studied by several authors, for instance Dopita et al. (1997), Reyes et al. (1997), and Richer (1993). To date, there are only few other galaxies. In fact, abundance analysis requires the determination of electron temperature and density, as well as the intensities of various lines of different ionization states. The most important line for temperature determination, [O III] $4363 \AA$ is, for instance, from 50 to 200 times weaker than [O III] $5007 \AA$ (e.g. Ford et al. 2002). Before our work, the farthest galaxy with direct abundance measurements in PNe was M 31 (750 kpc, Freedman et al. 2001). Chemical abundances of PNe

Send offprint requests to: L. Magrini

e-mail: laura@arcetri.astro.it

* Based on observations obtained at the $4.2 \mathrm{~m}$ WHT telescope operated on the island of La Palma by the Isaac Newton Group in the Spanish Observatorio del Roque de Los Muchachos of the Instituto de Astrofisica de Canarias. in M 31 have been investigated by Jacoby \& Ciardullo (1999, hereafter JC99) and Stasinska et al. (1998), as well as by Hyung et al. (2000) who used the data from the previous work of Stasinska and collaborators. A total of $45 \mathrm{PNe}$ have been studied. In the companion galaxies of M 31, spectrophotometric data have been obtained for PNe of NGC 185, NGC 205 (Richer \& McCall 1995) and of M 32 (Stasinska et al. 1998; Hyung et al. 2000). In the Fornax galaxy, one PN is known and its chemical abundances have been derived by Danziger et al. (1978) and Walsh et al. (1997). Spectrophotometry of the two PNe in the Sagittarius dwarf elliptical galaxy was done by Walsh et al. (1997). Chemical abundances of PNe in NGC 6822 were computed by Richer \& McCall (1995). Spectra of $5 \mathrm{PNe}$ in the giant elliptical galaxy NGC 5128 (Centaurus A) at the distance of 3.5 Mpc were analyzed by Walsh et al. (1999). These are the farthest PNe where chemical abundances have been measured. This was done using diagnostic line ratios of various ions without, however, a direct determination of the electron temperature.

The galaxy M 33 (NGC 598) is the third-brightest member of the Local Group. Its large angular size (optical size $53^{\prime} \times 83^{\prime}$, Holmberg 1958) and its intermediate inclination $i=56^{\circ}$ (Zaritsky 1999) make it particularly suitable for studies of spiral structure and stellar content (van den Bergh 2000). A total of 131 candidate PNe are known in M 33 distributed throughout the whole galaxy (Magrini et al. 2000, 2001a). The objects were identified with the following two criteria: $i$ ) they should appear both in the [O III] and $\mathrm{H} \alpha+[\mathrm{N}$ II] images but not in the continuum frame, and ii) they should have a stellar point spread function. In this paper, we present spectroscopy 
of 39 PN candidates and of 9 unclassified emission-line objects discovered in our previous survey (Magrini et al. 2000, hereafter M00). In Sect. 2 we describe the observations, the reduction procedures and the flux measurement with their dereddening. The analysis of the spectra and the determination of the physical and chemical properties of the PNe are presented in Sect. 3. A critical discussion on the chemical abundances is given in Sect. 4. Conclusions follow in Sect. 5.

\section{Observations and data analysis}

Thirty-nine candidate PNe and nine unclassified objects with emission lines and a non-negligible continuum, selected from the list of M00, were observed on October 16-17, 2001. We have used AF2/WYFFOS, the multi-object, wide field, fibre spectrograph working at the prime focus of the $4.2 \mathrm{~m}$ William Herschel Telescope (La Palma, Spain). The WYFFOS spectrograph was used with a single setup: the R600B grating (600 line $\mathrm{mm}^{-1}$ ) providing a dispersion of $3.0 \AA /$ pixel. The resulting spectral range, from $4300 \AA$ to $7380 \AA$, included the basic lines needed for the classification of the objects as $\mathrm{PNe}$ and the determination of their chemical abundances. However, due to the arrangement of fibres at the spectrograph entrance, not all the spectra start at the same wavelength and for $15 \mathrm{PNe}$ the $\lambda 4363 \AA$ spectral region was not observed. The spectrograph WYFFOS was equipped with a $1024 \times 1024$ TEK CCD. We used the Small Fibre module which is made of 150 science fibres with 1.6 arcsec diameter $(90 \mu \mathrm{m})$ projected on the sky.

Our targets, their astrometry and their absolute [O III] $5007 \AA$ and $\mathrm{H} \alpha+[\mathrm{N}$ II] fluxes came from our previous INT+WFC images (M00, Magrini et al. 2001a). The accuracy in their positions is better than 0.5 arcsec rms. Our set of targets was chosen among the PNe spanning a range of 3 mag in [O III] $\lambda 5007 \AA$ from the bright cutoff of their luminosity function, and included also several of the unclassified objects of our sample, i.e. emission-line objects with a non-negligible continuum emission (M00). Targets are distributed rather uniformly over the face of the galaxy (Fig. 1). We used the remaining fibres to take simultaneous sky/background spectra. A total of 13 science exposures of $2400 \mathrm{~s}$ each were taken ( 5 in the first night and 8 in the second night) through light cirrus with 1.5 arcsec seeing.

Several offset sky exposures using the same fibre configuration were taken before and after the M 33 observations in order to do a correct sky subtraction.

\subsection{Data reduction}

The data were reduced using the IRAF multi-fibre spectra reduction package DOFIBER. The sky subtraction represented the most difficult part of the reduction. As already mentioned, we used some of the fibres to monitor the sky background during the science exposures and we also had several offset sky exposures with the same fibre configuration. We used the sky fibres in our exposures of M 33 to monitor the relative intensity of the atmospheric emission lines, which are known to vary during the night. However, these sky fibres lie in different positions in the spectrograph focal plane and consequently produce spectra with different spatial and spectral instrumental profiles. For this reason, they cannot be used for a direct sky subtraction. There are instead no variations in the instrumental profiles for each individual fibre between the offset sky frame spectra and the science frames, as fibres are fixed at the spectrograph entrance. We therefore used these offset sky spectra to do the sky subtraction, after correcting the relative intensity of the atmospheric lines using the mean sky spectrum computed from the sky fibres observed at the same time as the science targets.

\subsection{Flux calibration}

Relative data flux calibration was obtained taking spectra with several fibres of the spectrophotometric standard stars G191B2B (Oke 1990) and 40 Eri B (Oke 1974). Using AF2, the absolute flux calibration cannot be done, since one is limited by the fixed fibre diameter. In addition, it is impracticable to observe a spectrophotometric standard star in all the science fibres in order to have a sensitivity function for each fibre. In order to test whether a mean sensitivity function can be used for all the fibres, the standard star G191B2B was observed in nine fibres and the sensitivity functions of those fibres were analyzed. The variations of the spectral response of each fibre are within $\sim 1 \%$ in the whole spectral range, as shown in Fig. 2. This result allows us to use a mean sensitivity function for all the fibres.

\subsection{Flux measurement and de-reddening}

Emission line fluxes of the spectra were measured using the MIDAS/ALICE package. We also used the IRAF task TWOFITLINES (IACTASKS, Acosta 1999) to estimate errors on the fluxes. This task computes the uncertainty of model parameters by a number of repeated fits to simulated data, generated from the best model with random noise added. The absolute errors on the measurement of the emission-line strengths are given; percentage errors (not including flux calibration errors) on the $\mathrm{H} \beta$ line are shown in Table 1. The background noise and the sky subtraction are the greatest sources of error and they affect uniformly the whole spectral range where emission lines were measured. Thus, the absolute error of each emission line of our spectra is approximately constant and equal to the absolute error of $\mathrm{H} \beta$ line. Moreover, systematic errors due to the flux calibration, including the errors on the Oke fluxes $(\sim 0.01 \mathrm{mag})$, were estimated to be approximately $5-10 \%$ along the whole spectral range.

The observed line fluxes have been corrected for the effect of the interstellar extinction. The extinction law of Mathis (1990) with $R_{V}=3.1$ has been used. The $c_{\beta}$, which is the logarithmic difference between the observed and unreddened $\mathrm{H} \beta$ fluxes, was determined comparing the observed Balmer $I(\mathrm{H} \alpha) / I(\mathrm{H} \beta)$ ratio with its theoretical value. All fluxes are presented on a scale relative to $\mathrm{H} \beta$, where $F(\mathrm{H} \beta)$ is set to 100 . Observed fluxes and $c_{\beta}$ for the objects in whose spectra we could extract information are shown in Table 1 . The percentage errors on the $\mathrm{H} \beta$ fluxes are presented in the last line of Table 1. 


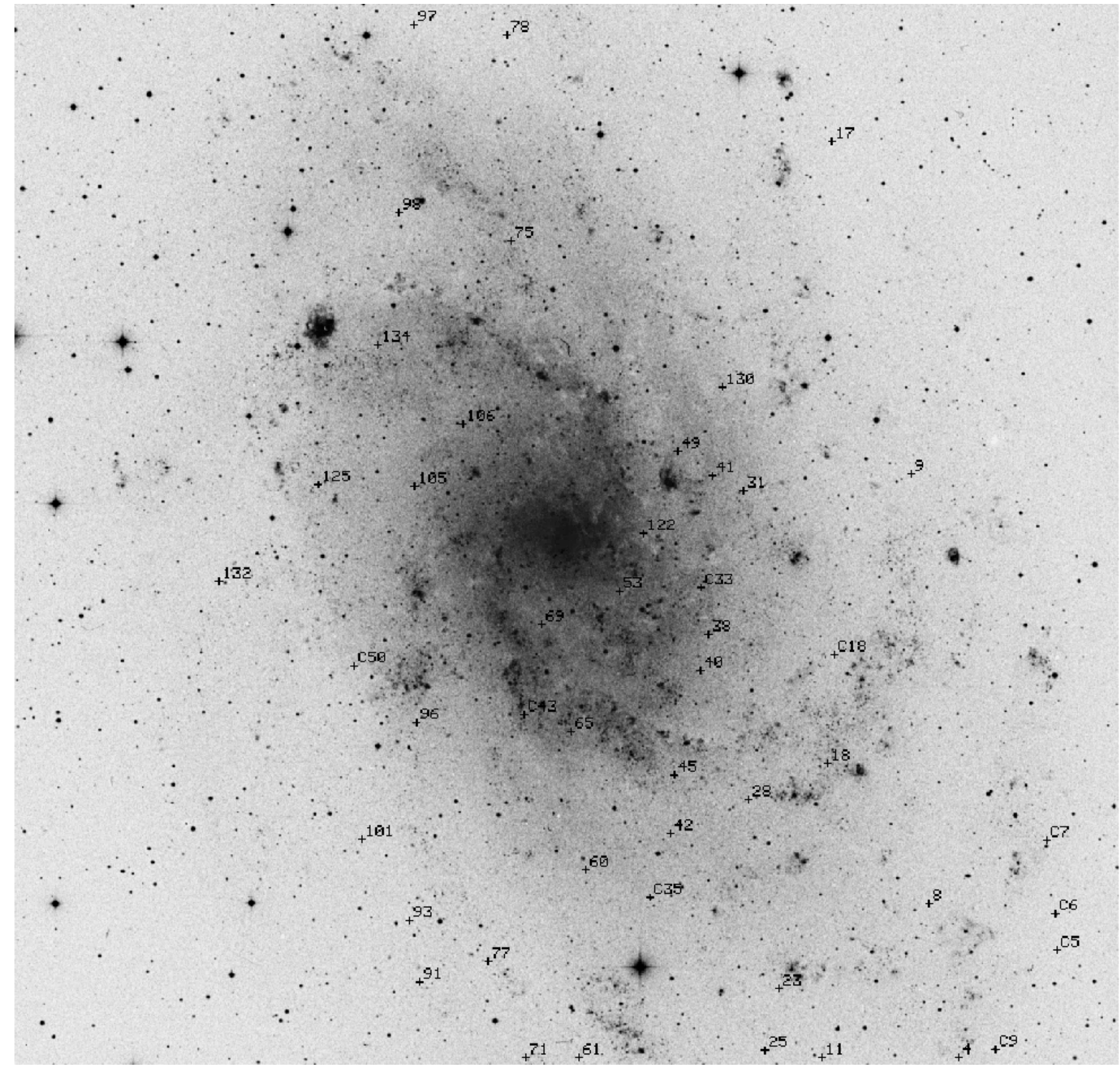

Fig. 1. Target positions in the M 33 galaxy. The i.d. numbers are from M00. The emission-line + continuum objects are shown with their i.d. number, preceded by a capital C. The image is from the ESO-Digitized Sky Survey and its size is $40^{\prime} \times 40^{\prime}$. North is at the top, East to the left.

\section{Analysis of the spectra}

Out of the 48 targets observed, the spectra of 3 candidate PNe and 2 unclassified objects were so weak that no usable information could be extracted, whereas one of the unclassified objects, namely MCMPc9, turned out to be a normal star. The nature and properties of the remaining 42 objects are discussed in the following.

\subsection{The Balmer decrement: PNe extinction}

The weighted mean value of the logarithmic extinction, $c_{\beta}$, of the PN candidates (Table 1 ) is $0.73 \pm 0.21$. Approximately $30 \%$ of the candidates have a very small $c_{\beta}$ and presumably they lie in front of the galaxy. The other PNe have $c_{\beta}$ greater than 0.5: they lie on the far side of M 33 and/or in regions of the spiral arms where the extinction is higher. The mean logarithmic extinction of our disc PNe is similar to that found by JC99 in three PNe on the disc of M 31 ( $c_{\beta}=0.50$; JC99).

\subsection{Diagnostic diagrams}

Magrini et al. (2000) introduced the excitation parameter $R=$ $\frac{[\mathrm{OIII}]}{\mathrm{H} \alpha+[\mathrm{NII}]}$ as a crude indicator of the nature of extragalactic emission line objects found in narrow-band surveys, arguing that most objects with $R>1$ (and point-like at the distance of M 31 and M 33) are expected to be PNe. Ciardullo et al. (2002) suggest a slightly higher limit $(R>1.6)$ for PN candidates in the brightest 1-mag bin of the PNLF. Nevertheless, using $R>1.6$, 
Table 1. Observed line fluxes.

\begin{tabular}{|c|c|c|c|c|c|c|c|c|}
\hline \multirow[t]{2}{*}{ Identification } & & MCMP8 & MCMP9 & MCMP17 & MCMP18 & MCMP23 & MCMP25 & MCMP28 \\
\hline & & PN & PN & - & PN & $\mathrm{H}_{\text {II } \mathrm{r}}$ & PN & PN \\
\hline 4685.7 & $\mathrm{He}_{\text {II }}$ & - & - & - & - & - & - & 29.1 \\
\hline 4861.3 & $\mathrm{H} \beta$ & 100. & 100. & 100. & 100. & 100. & 100. & 100. \\
\hline 4958.9 & [O III] & 160. & 467. & 206. & 255. & 67.3 & 221. & 396. \\
\hline 5006.8 & [O III] & 454. & 1275 . & 469. & 641. & 165. & 803. & 1198. \\
\hline 5875.7 & $\mathrm{He}_{\mathrm{I}}$ & - & 33.9 & - & - & 22.0 & - & 13.6 \\
\hline 6562.8 & $\mathrm{H} \alpha$ & 636. & 741. & 285. & 350. & 364. & 446. & 509. \\
\hline 6583.4 & {$[\mathrm{~N}$ II] } & 52.3 & 108. & - & 75.3 & 29.8 & 61.0 & 45.3 \\
\hline 6678.1 & $\mathrm{He}_{\mathrm{I}}$ & 14.8 & 20.8 & - & 20.3 & - & - & 11.5 \\
\hline 6716.5 & {$[\mathrm{~S}$ II] } & 23.9 & 17.5 & 18.3 & 65.0 & 70.6 & 132. & 50.2 \\
\hline 6730.8 & [S II] & 23.9 & 21.6 & 18.8 & 39.2 & 50. & - & 36.6 \\
\hline 7065.3 & He I & 23.1 & - & - & 35.0 & - & - & - \\
\hline 7135.8 & Ar III & 62.7 & 80.2 & - & 62.8 & - & - & 48.9 \\
\hline 7325 (*) & [O II] & 22.7 & 15.2 & - & - & - & - & 19.0 \\
\hline$c_{\beta}$ & & 1.15 & 1.36 & 0.00 & 0.30 & 0.35 & 0.64 & 0.83 \\
\hline \multirow[t]{3}{*}{$\mathrm{H} \beta$ error } & & $2 \%$ & $10 \%$ & $15 \%$ & $4 \%$ & $8 \%$ & $50 \%$ & $6 \%$ \\
\hline & & MCMP31 & MCMP38 & MCMP40 & MCMP41 & MCMP42 & MCMP45 & MCMP49 \\
\hline & & $\mathrm{H}$ І $\mathrm{r} / \mathrm{PN}$ & $\mathrm{PN}$ & $\mathrm{PN}$ & SNR & SNR & PN & $\mathrm{PN}$ \\
\hline 4685.7 & He II & 13.6 & - & - & 30.6 & - & - & 16.5 \\
\hline 4861.3 & $\mathrm{H} \beta$ & 100. & 100. & 100. & 100. & 100. & 100. & 100. \\
\hline 4958.9 & [O III] & 197. & 134. & 517. & 119. & 339. & 218. & 208. \\
\hline 5006.8 & [O III] & 314. & 158. & 980. & 265. & 840. & 423. & 487. \\
\hline 5875.7 & $\mathrm{He}_{\mathrm{I}}$ & 15.4 & 91.9 & - & 64.6 & - & 14.6 & 21.4 \\
\hline 6562.8 & $\mathrm{H} \alpha$ & 288. & 285. & 662. & 736. & 329. & 296. & 595. \\
\hline 6583.4 & {$[\mathrm{~N}$ II] } & 103. & 71.9 & 964. & 250. & 130. & 96.1 & 88.8 \\
\hline 6678.1 & He I & 13.3 & - & - & - & - & - & \\
\hline 6716.5 & {$[\mathrm{~S}$ II] } & 41.0 & 28.4 & 183. & 189. & 284. & 26.6 & 53.0 \\
\hline 6730.8 & [S II] & 30.7 & - & 135. & 158. & 123. & 23.6 & 32.7 \\
\hline 7135.8 & Ar III & 32.6 & - & - & 75.1 & - & 15.9 & - \\
\hline$c_{\beta}$ & & 0.01 & 0.00 & 1.20 & 1.36 & 0.20 & 0.05 & 1.05 \\
\hline \multirow[t]{3}{*}{$\mathrm{H} \beta$ error } & & $8 \%$ & $15 \%$ & $40 \%$ & $20 \%$ & $50 \%$ & $10 \%$ & $8 \%$ \\
\hline & & MCMP53 & MCMP60 & MCMP61 & MCMP65 & MCMP68 & МСMP69 & MCMP71 \\
\hline & & SNR & PN & $\mathrm{H}$ II r/PN & PN & PN & PN & $\mathrm{PN}$ \\
\hline 4363.2 & {$[\mathrm{O}$ III] } & - & 16.4 & - & 20.2 & - & - & - \\
\hline 4685.7 & He II & - & - & 78.2 & - & - & - & - \\
\hline 4740.2 & Ar IV & - & - & - & - & - & 13.4 & - \\
\hline 4861.3 & $\mathrm{H} \beta$ & 100. & 100. & 100. & 100. & 100. & 100. & 100. \\
\hline 4958.9 & [O III] & - & 568. & 274. & 510. & 160. & 288. & 365 . \\
\hline 5006.8 & [O III] & - & 1475. & 637. & 1259. & 520. & 748. & 553. \\
\hline 5875.7 & $\mathrm{He}_{\mathrm{I}}$ & - & - & - & - & 32.4 & 41.9 & - \\
\hline 6562.8 & $\mathrm{H} \alpha$ & 1177. & 484. & 320. & 567. & 365. & 555. & 658. \\
\hline 6583.4 & {$[\mathrm{~N}$ II] } & 428. & 221. & 98.1 & 163. & 134. & 693. & 539. \\
\hline 6716.5 & {$[\mathrm{~S}$ II] } & 410. & 54.1 & 43.3 & 71.7 & 67.2 & 201. & 53.7 \\
\hline 6730.8 & {$[\mathrm{~S}$ II] } & 356. & 48.6 & 49.7 & 74.8 & 63.3 & 163. & 63.0 \\
\hline 7065.3 & $\mathrm{He}_{\mathrm{I}}$ & - & - & - & 22.5 & 16.2 & 68.2 & - \\
\hline 7135.8 & Ar III & - & 52.0 & 84.8 & 119. & 39.1 & 145. & - \\
\hline $7325\left(^{*}\right)$ & [O пा] & - & 29.6 & - & 40. & - & - & - \\
\hline$c_{\beta}$ & & 2.03 & 0.76 & 0.17 & 0.97 & 0.35 & 0.95 & 1.19 \\
\hline $\mathrm{H} \beta$ error & & $50 \%$ & $20 \%$ & $20 \%$ & $10 \%$ & $8 \%$ & $20 \%$ & $50 \%$ \\
\hline
\end{tabular}

Note: $(*)$ Sum of the [O пा] doublet lines at 7319.6 and $7330.2 \AA$.

we would exclude a large number of low excitation PNe. In fact about $40 \%$ of confirmed Galactic PNe have $R<1.6$ (see M00, Fig. 3). In the following analysis on the nature of candidate $\mathrm{PNe}$, we consider adequate for PNe values of $R$ from 0.3 to larger than 1.6, as expected for a "normal" (i.e. Galactic) population of PNe. However, to identify bona fide PNe, more precise indicators should be used when, as in our case, a larger part of the spectrum is available. The empirical emission line diagrams (Sabbadin \& D'Odorico 1976; García-Lario et al. 1991; Riesgo-Tirado \& López 2002) have proved to be very useful in distinguishing among different classes of Galactic ionized nebulae, the most effective one being probably that displaying the $\mathrm{H} \alpha /\left[\mathrm{N}\right.$ II] vs. $\mathrm{H} \alpha /\left[\mathrm{S}_{\mathrm{II}}\right]$ line ratios, as it neatly separates $\mathrm{PNe}$ from $\mathrm{H}$ II regions and supernova remnants. This diagnostic diagram is especially valuable for extragalactic objects since it involves relatively strong lines and avoids information less easily obtainable in the UV or IR spectral ranges. We have recalculated the PNe zone in this diagram using the most precise Galactic and extragalactic data available: Kingsburgh \& Barlow (1994, hereafter KB94), Kaler et al. (1996), Cuisinier et al. (2000) for Galactic PNe; de Freitas Pacheco et al. (1993), Vassiliadis et al. (1992) and Stanghellini et al. (2002) for LMC and SMC; JC99 and Stasinska et al. (1998) for M 31; Walsh et al. (1997) for Fornax. Note that the new limits shift the upper border of the PNe band more into the $\mathrm{H}$ II region box. 
Table 1. continued.

\begin{tabular}{|c|c|c|c|c|c|c|c|c|}
\hline \multirow{2}{*}{ Identification } & & MCMP75 & MCMP77 & MCMP78 & MCMP91 & MCMP93 & MCMP96 & $\overline{\mathrm{MCMP} 101}$ \\
\hline & & PN & - & $\mathrm{H}_{\text {II } \mathrm{r}}$ & PN & PN & PN & PN \\
\hline 4363.2 & [O III] & - & - & - & 9.6 & 6.09 & - & 10.0 \\
\hline 4471.5 & Не пा & - & - & 7.53 & 12.3 & 14.7 & - & - \\
\hline 4685.7 & Не п & - & - & - & 18.8 & 8.52 & - & - \\
\hline 4740.2 & Ar IV & 11.9 & - & - & - & - & - & - \\
\hline 4861.3 & $\mathrm{H} \beta$ & 100. & 100. & 100. & 100. & 100. & 100. & 100. \\
\hline 4958.9 & [O III] & 451. & 265. & 51.1 & 448. & 406. & 404. & 460. \\
\hline 5006.8 & {$[\mathrm{O}$ ІाI] } & 1212. & 765. & 157. & 1348. & 1200. & 1199. & 1385. \\
\hline 5875.7 & He I & - & - & 23.9 & - & - & 19.9 & 24.3 \\
\hline 6562.8 & $\mathrm{H} \alpha$ & 366. & 600. & 427. & 433. & 430. & 438. & 499. \\
\hline 6583.4 & {$[\mathrm{~N} \mathrm{II]}$} & 151. & - & 73.2 & 153. & 91.8 & 96.6 & 74.1 \\
\hline 6678.1 & He I & 17.3 & - & 8.66 & 11.0 & 5.95 & 10.1 & 11.4 \\
\hline 6716.5 & {$[\mathrm{~S} \mathrm{II]}$} & 10.4 & - & 50.1 & 9.4 & 19.5 & 33.0 & 15.2 \\
\hline 6730.8 & {$[\mathrm{~S}$ II] } & 18.7 & - & 35.2 & 13.2 & 21.6 & 22.5 & 11.3 \\
\hline 7065.3 & He I & 24.6 & - & 7.32 & 10.5 & - & 23. & - \\
\hline 7135.8 & Ar III & 45.3 & - & 23.7 & 31.2 & 27.3 & 36.5 & 38.6 \\
\hline 7319.6 & {$\left[\begin{array}{ll}\mathrm{O} & \text { II] }\end{array}\right.$} & - & - & 12.1 & 10.5 & 10.8 & - & 12.3 \\
\hline 7330.2 & {$[\mathrm{O} \mathrm{II]}$} & - & - & 10.9 & 7.7 & 8.3 & - & 10.8 \\
\hline$c_{\beta}$ & & 0.36 & 1.06 & 0.58 & 0.60 & 0.59 & 0.62 & 0.80 \\
\hline \multirow[t]{3}{*}{$\mathrm{H} \beta$ error } & & $8 \%$ & $50 \%$ & $9 \%$ & $2 \%$ & $5 \%$ & $5 \%$ & $3 \%$ \\
\hline & & MCMP105 & MCMP106 & MCMP122 & MCMP125 & MCMP127 & MCMP130 & MCMP132 \\
\hline & & PN & PN & $\mathrm{H}$ II r $/ \mathrm{PN}$ & PN & $\mathrm{H}$ II $\mathrm{r}$ & - & $\mathrm{H}_{\text {II r } \mathrm{r} / \mathrm{PN}}$ \\
\hline 4363.2 & [O III] & - & - & 1.36 & - & - & - & - \\
\hline 4471.5 & Не пा & - & - & 8.68 & - & - & 20.5 & 7.93 \\
\hline 4861.3 & $\mathrm{H} \beta$ & 100. & 100. & 100. & 100. & 100. & 100. & 100. \\
\hline 4958.9 & [O III] & 367. & 228 & 58.0 & 189. & 61.8 & 50.9 & 90.8 \\
\hline 5006.8 & {$[\mathrm{O}$ шII] } & 787. & 265. & 179. & 583. & 194. & 124. & 234. \\
\hline 5875.7 & $\mathrm{He}$ I & - & - & 25.4 & 14.9 & 27.0 & 18.0 & 23.5 \\
\hline 6562.8 & $\mathrm{H} \alpha$ & 382. & 785. & 689. & 458. & 755. & 395. & 621. \\
\hline 6583.4 & {$[\mathrm{~N} \mathrm{II]}$} & 43.6 & 476. & 151. & 123. & 221. & 160. & 88.7 \\
\hline 6678.1 & $\mathrm{He}$ I & - & - & 10.9 & - & 77.3 & - & 21.0 \\
\hline 6716.5 & {$[\mathrm{~S}$ ㅍ] } & 18.2 & 81.3 . & 61.6 & 53.8 & 148. & - & 40.8 \\
\hline 6730.8 & {$[\mathrm{~S}$ пI] } & - & 55.1 & 55.8 & 35.9 & 119. & - & 36.9 \\
\hline 7065.3 & $\mathrm{He}_{\mathrm{I}}$ & - & - & 10.5 & - & - & - & 21.7 \\
\hline 7135.8 & Ar III & 15.3 & - & 29.7 & 34.1 & 95.5 & - & 50.4 \\
\hline 7319.6 & [O II] & - & - & 10.0 & - & - & - & - \\
\hline 7330.2 & {$[\mathrm{O}$ II] } & - & - & 10.3 & $26.9^{*}$ & - & - & - \\
\hline$c_{\beta}$ & & 0.42 & 1.45 & 1.26 & 0.68 & 1.39 & 0.47 & 1.11 \\
\hline \multirow[t]{3}{*}{$\mathrm{H} \beta$ error } & & $12 \%$ & $40 \%$ & $2 \%$ & $5 \%$ & $12 \%$ & $10 \%$ & $4 \%$ \\
\hline & & MCMP134 & MCMPc5 & MCMPc6 & MCMPc18 & MCMPc35 & MCMPc43 & 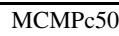 \\
\hline & & PN & $\mathrm{H} \mathrm{II} \mathrm{r}$ & $\mathrm{H}$ II $\mathrm{r}$ & SNR & $\mathrm{H}$ II $\mathrm{r}$ & SNR & - \\
\hline 4685.7 & Не п г & 28.5 & - & - & - & - & - & - \\
\hline 4861.3 & $\mathrm{H} \beta$ & 100. & 100. & 100. & 100. & 100. & 100. & 100. \\
\hline 4958.9 & {$[\mathrm{O}$ шII] } & 270. & - & 27.4 & - & 21.5 & 23.0 & - \\
\hline 5006.8 & {$[\mathrm{O}$ III] } & 703. & 33.6 & 101. & - & 72.0 & 30.3 & 9.73 \\
\hline 5875.7 & He I & - & - & 7.14 & - & 9.90 & - & - \\
\hline 6562.8 & $\mathrm{H} \alpha$ & 490. & 632. & 473. & 371. & 476. & 394. & 738. \\
\hline 6583.4 & {$[\mathrm{~N}$ II] } & 173. & 78.6 & 49.4 & 55.8 & 97.8 & 174. & - \\
\hline 6678.1 & $\mathrm{He}$ I & 32.2 & - & 3.90 & - & 5.62 & - & - \\
\hline 6716.5 & {$[\mathrm{~S}$ пI] } & 60.6 & 96.7 & 42.7 & 136. & 41.2 & 147. & - \\
\hline 6730.8 & {$[\mathrm{~S}$ I] } & 51.2 & 56.4 & 29.2 & 99.0 & 29.0 & 110 & - \\
\hline 7065.3 & $\mathrm{He} \mathrm{I}$ & - & - & 5.13 & - & 4.93 & - & 18.8 \\
\hline 7135.8 & Ar III & 64.7 & - & 7.94 & - & 10.1 & - & - \\
\hline$c_{\beta}$ & & 0.78 & 1.14 & 0.73 & 0.38 & 0.73 & 0.46 & 1.36 \\
\hline $\mathrm{H} \beta$ error & & $20 \%$ & $10 \%$ & $1 \%$ & $25 \%$ & $0.3 \%$ & $7 \%$ & $3 \%$ \\
\hline
\end{tabular}

The M 33 PN candidates were put in this diagram (Fig. 3) coded by their $R$ parameter. In this way we supplement the diagram with the information of the [O III] line which is the most important optical line characterizing a PN. We analyze in the following the location of all objects in this diagram. Five of the nine objects with non-negligible continuum (see M00) have good data for the involved lines (Table 1). Two of them (MCMPc18 and 43) are near the SNRs locus; both have strong [S II] lines (in MCMPc18 the [S II] lines are stronger than $\left[\mathrm{N}_{\mathrm{II}}\right]$ ). The remaining three objects appear in the $\mathrm{H}$ II region area. All of them are associated with a low value of the excitation indicator $(R<0.5)$. Only one is located in the overlapping area between the $\mathrm{PNe}$ and the $\mathrm{H}$ II regions (MCMPc35) but it is indeed a low excitation object and thus presumably it is not a planetary nebula. Most objects without continuum were found to occupy the PNe region 


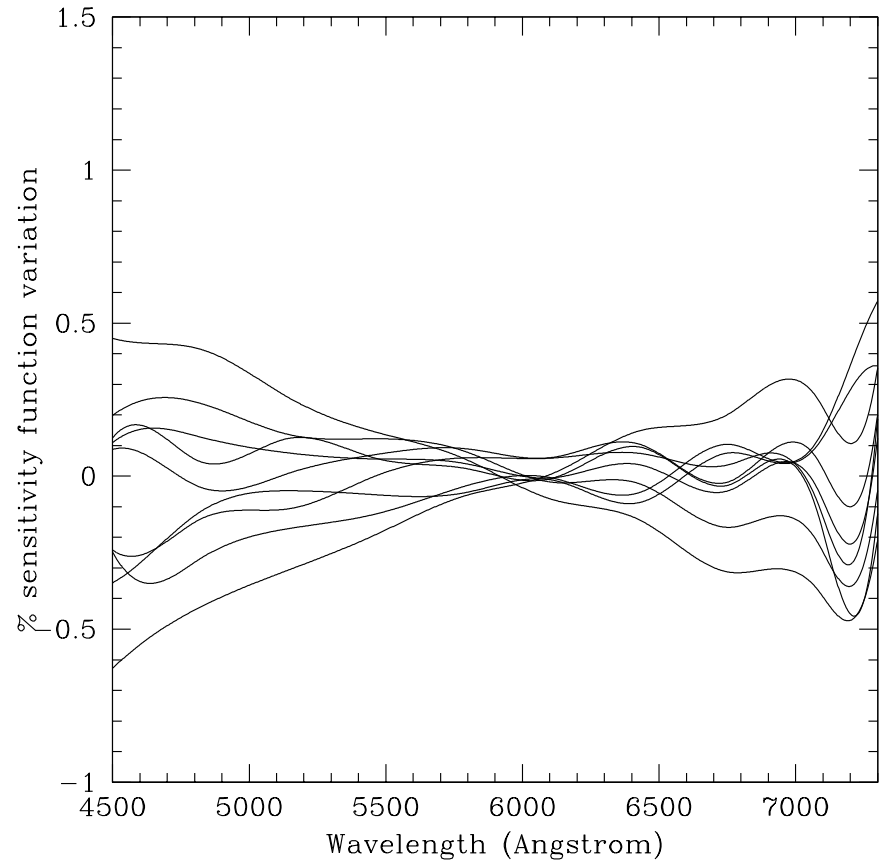

Fig. 2. The percentage variation from fibre to fibre of the sensitivity function.

although they appear slightly displaced towards the upper border of the PNe band. Note, on the other hand, that the three objects where chemical abundances were determined (Sect. 4) lie inside the PNe area, and that all candidates in the PNe area show values of $R$ from 0.3 to larger than 1.6, as expected for a normal population of PNe. In fact, only three candidates in the overlapping $\mathrm{PNe}$ and $\mathrm{H}$ II region area have $0.5<R<1$ (MCMP31, MCMP122, MCMP132). Although those objects can be misidentified with $\mathrm{H}$ II regions, they can equally well be low excitation PNe. The remaining candidates can be considered bona fide planetary nebulae. A total of ten candidates lie above the line plotted to mark the PNe region: seven in the $\mathrm{H}$ II region area and three in the SNR area. Three of the former, MCMP23, MCMP78 and MCMP127, have $R<0.5$ and are probably unresolved $\mathrm{H}$ II regions. MCMP61 has higher excitation and its nature remains doubtful. The other three objects (MCMP18, MCMP25, MCMP28) have a high $R$ and lie near the PNe locus where there are also numerous confirmed PNe. They are probably genuine PNe. MCMP41, MCMP42 and MCMP53 occupy the SNRs locus, each with different excitation.

Concluding, no emission line object with some continuum emission (called "unclassified" in our previous work; M00) can be considered to be $\mathrm{PNe}$, whereas most of our candidate $\mathrm{PNe}$ (objects without continuum) are shown to occupy the same locus in the $\mathrm{H} \alpha /[\mathrm{N}$ II] vs. $\mathrm{H} \alpha /[\mathrm{S}$ II] diagnostic diagram as Galactic and extragalactic PNe. Three candidates which lie in the upper $\mathrm{H}$ II region area and have a low value of $R$ are rejected as PNe, whereas four objects in that area showing high $R$ are classified as PNe. Three candidates located in the SNRs region are rejected as $\mathrm{PNe}$ and another three candidates in the overlapping area between $\mathrm{PNe}$ and $\mathrm{H}$ II regions can be low excitation $\mathrm{PNe}$ or compact $\mathrm{H}$ II regions. All in all, $72 \%$ of our

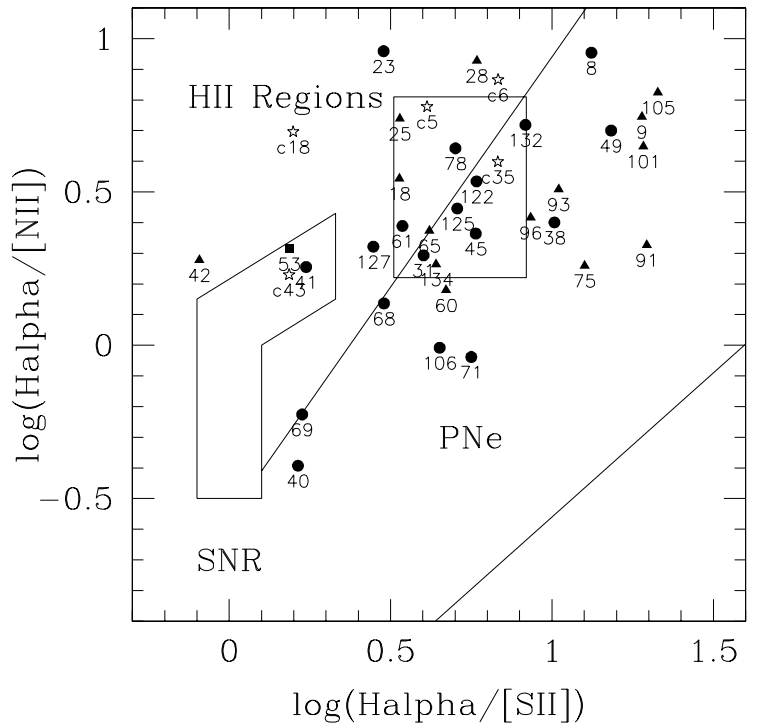

Fig. 3. Diagnostic diagram (originally from Sabbadin \& D'Odorico 1976). The Galactic H II regions and SNRs areas are from GarcíaLario et al. 1991. The PNe region is drawn using recent data of Galactic and extragalactic PNe (see text). Our candidate PNe are represented by triangles $(R>1.6)$, circles $(0.3<R<1.6)$ and squares $(R<0.3)$. The emission-line objects with non-negligible continuum are represented by stars.

candidate $\mathrm{PNe}$ with good spectral data (i.e. with $\mathrm{H} \alpha$, [N II], [S II], [O III] lines well measured) are bona fide PNe; the remaining $28 \%$ will include $\mathrm{H}$ II regions, SNR and low excitation PNe. From the galaxy distribution of $R$ discussed in our previous work (M00), we did expect a contamination by $\mathrm{H}$ II regions of approximately $11 \%$, a figure which is in rough agreement with the values found above. The probable nature of each object, as deduced from the preceding discussion, is specified in Table 1.

\subsection{Electron density and temperature}

The essential line for electron temperature determination, $4363 \AA$ [O III], was measured in 6 PNe (MCMP60, 65, 91, $93,101,122)$. For the other 36 objects, it was not observed: for 21 objects it was too faint to be detected while in the remaining 15 the spectral range did not include it (Sect. 2). The spectra of PNe MCMP91, 93, 101 and 122 had good signal to noise ratio, whereas MCMP60 and 65 were quite noisy. To test the quality of the spectra of the PNe where physical and chemical quantities can be computed, we compared the observed and theoretical values for the line ratios set by atomic constants and present in our spectral range: [O III] $I(5007) / I(4959)$ and $\left[\mathrm{N}_{\mathrm{II}}\right] \mathrm{I}(6584) / I(6548)$. The observed mean for the [O III] ratio is $2.85 \pm 0.40$ and for the [N $\mathrm{N}$ ] ratio is $2.85 \pm 0.50$, considering the sample of six nebulae, and $3.01 \pm 0.10$ and $3.02 \pm 0.30$ for MCMP91, 93, 101 and 122, whereas the theoretical value, corrected for the energy of the levels, are respectively 2.96 and 2.98 (Osterbrock 1989). We computed electron temperature and density for the whole six nebulae sample, avoiding chemical abundance determinations for the two $\mathrm{PNe}$ whose spectra were noisier, as deduced from their observed [O III] 
Table 2. Electron densities and temperatures.

\begin{tabular}{llll}
\hline \hline & MCMP60 & MCMP 65 & MCMP 91 \\
\hline$n_{\mathrm{e}}\left(\mathrm{cm}^{-3}\right)$ & 300 & 700 & 1900 \\
$T_{[\mathrm{OIII}]}(\mathrm{K})$ & 12600 & 14900 & 11100 \\
$d(\mathrm{kpc})$ & 3.1 & 1.8 & 4.3 \\
$\operatorname{mag}_{[\text {[IIII] }}$ & 22.50 & 21.98 & 20.78 \\
$R$ & 3.2 & 3.2 & 3.2 \\
& & & \\
\hline & MCMP93 & MCMP101 & MCMP122 \\
\hline$n_{\mathrm{e}}\left(\mathrm{cm}^{-3}\right)$ & 850 & 100 & 400 \\
$T_{[\text {OIII] }}(\mathrm{K})$ & 9900 & 11500 & 13200 \\
$d(\mathrm{kpc})$ & 3.8 & 3.4 & 0.8 \\
$\operatorname{mag}_{[\text {OIII] }]}$ & 21.64 & 21.19 & 21.30 \\
$R$ & 3.2 & 4.1 & 0.5 \\
\hline
\end{tabular}

and $\left[\mathrm{N}_{\mathrm{II}}\right]$ line ratios and from their overall low $\mathrm{S} / \mathrm{N}$ and taking out also the object MCMP122 whose classification as a PN was doubtful.

Electron temperatures and densities have been derived using the ratios [O III] $I(5007) / I(4363)$ for $T_{\mathrm{e}}$ and $\left[\begin{array}{ll}\mathrm{S} & \mathrm{II}\end{array}\right]$ $I(6717) / I(6731)$ for $n_{\mathrm{e}}$. The most relevant source of error for the temperature-sensitive ratio is the error associated with the weak [O III] $4363 \AA$ line, whereas the error on the density ratio is based on the accuracy of the fluxes of both lines of the [S II] doublet. The uncertainty on $T_{\mathrm{e}}$ is approximately $15 \%$ for the $\operatorname{good} \mathrm{S} / \mathrm{N}$ spectra, and somewhat higher for the remaining ones. Errors in the electron density are larger but density essentially does not enter into the chemical abundance determinations. We adopted $T_{\mathrm{e}}[\mathrm{N}$ II $]=T_{\mathrm{e}}[\mathrm{O} \mathrm{III}]$, i.e. the same temperature for the high and low ionization ions, because the [N II] $5755 \AA$ line was not detected in our spectra and consequently $T_{\mathrm{e}}[\mathrm{N}$ II] could not be determined directly from the measurement of the [N $\mathrm{NI}]$ $I(5755) / I(6583)$ ratio. The nebular electron temperatures and densities for the 6 PNe where we could measure the [O III] $4363 \AA$ line are presented in Table 2 . In this Table their distances in kpc from the centre of $\mathrm{M} \mathrm{33}$, their $\operatorname{mag}_{\text {[OIII] }}$ (from M00, extinction corrected with the $c_{\beta}$ s of Table 1 ), and their de-reddened excitation parameter $R=\frac{[\mathrm{OIII}]}{\mathrm{H} \alpha+[\mathrm{NII}]}$ are also indicated.

\subsection{Chemical abundances}

As mentioned before, chemical abundances could be derived with a reasonable accuracy only for three out of the six PNe, namely MCPM91, 93, and 101, in whose spectra the [O III] $\lambda 4363 \AA$ was detected since their $\mathrm{S} / \mathrm{N}$ was good also for the weakest lines.

Helium ionic abundance have been computed considering the Case B recombination and using the effective recombination coefficients from Hummer \& Storey (1987) for $\mathrm{H} \beta$ and $\mathrm{He}^{2+}$ and from Brocklehurst (1971) for $\mathrm{He}^{+}$. The $\mathrm{He}$ I line strengths were corrected for the effect of collisional population of their upper state following Clegg (1987). The contribution of this effect is negligible in the case of low densities. The ionic
Table 3. Chemical abundances of three PNe in M 33.

\begin{tabular}{llll}
\hline \hline Ion/Elem. & MCMP91 & MCMP93 & MCMP101 \\
\hline $\mathrm{He}^{+} / \mathrm{H}$ & 0.17 & 0.09 & 0.14 \\
$\mathrm{He}^{2+} / \mathrm{H}$ & 0.02 & 0.01 & - \\
$\mathrm{He} / \mathrm{H}$ & 0.19 & 0.10 & 0.14 \\
$\mathrm{O}^{+} / \mathrm{H} \times 10^{4}$ & 1.44 & 2.37 & 2.96 \\
$\mathrm{O}^{2+} / \mathrm{H} \times 10^{4}$ & 3.25 & 4.20 & 3.03 \\
$i c f$ & 1.08 & 1.05 & 1.00 \\
$\mathrm{O} / \mathrm{H} \times 10^{4}$ & 5.06 & 6.92 & 5.72 \\
$\mathrm{~N}^{+} / \mathrm{H} \times 10^{5}$ & 1.90 & 1.22 & 0.77 \\
$i c f$ & 3.51 & 2.92 & 2.12 \\
$\mathrm{~N} / \mathrm{H} \times 10^{4}$ & 0.67 & 0.30 & 0.16 \\
$\mathrm{Ar}{ }^{2+} / \mathrm{H} \times 10^{6}$ & 1.49 & 1.51 & 1.48 \\
$i c f$ & 1.40 & 1.52 & 1.89 \\
$\mathrm{Ar} / \mathrm{H} \times 10^{6}$ & 2.08 & 2.29 & 2.80 \\
$\mathrm{~S}^{+} / \mathrm{H} \times 10^{6}$ & 0.49 & 0.85 & 0.36 \\
$i c f$ & 1.16 & 1.12 & 1.06 \\
$\mathrm{~S} / \mathrm{H} \times 10^{6}$ & 0.57 & 0.92 & 0.38 \\
\hline
\end{tabular}

metal abundances were derived from their collisionally excited lines solving the equations of statistical equilibrium. In order to calculate the total abundances, the unseen stages of ionization were accounted for using the standard ionization correction factors (ICF) (KB94). We used the same atomic data as KB94.

We estimated errors in the chemical abundances of these three objects on the bases of the error propagation across the procedure of evaluation of abundances, taking into account the uncertainties in the observed fluxes of the relevant lines and in the electron temperatures. Errors due to the uncertainties in the atomic quantities were not considered due to the extreme complexity of that, also regarding obvious compensation of these errors among the various atomic quantities. We obtained formal errors of: $20-30 \%$ for $\mathrm{He}, 25-50 \%$ for $\mathrm{O}, 40-70 \%$ for $\mathrm{N}$, and $30-70 \%$ for Ar. Errors are not given for $\mathrm{S}$ since its abundance is uncertain and it will be discussed in Sect. 4.2. The derived chemical abundances of the three PNe are listed in Table 3.

\section{Discussion on the chemical abundances}

\subsection{Comparison with other galaxies}

The median abundances of helium, nitrogen, oxygen, sulphur and argon of the three PNe are given in Table 4. He abundance is given as $\mathrm{He} / \mathrm{H}$, whereas $(\mathrm{N}, \mathrm{O}, \mathrm{S}, \mathrm{Ar})$ abundances are in the usual $\log \left[\frac{X}{\mathrm{H}}\right]+12$ units. In Table 4 we show for comparison chemical abundances of 15 PNe in M 31 (JC99), the Milky Way (from the sample of KB94), the LMC and the SMC (Clegg 1992), the Sagittarius dwarf galaxy (Walsh et al. 1997) and the elliptical galaxy Centaurus A (NGC 5128, Walsh et al. 1999). These data include all the most recent information concerning chemical abundances of extragalactic PNe (Ford et al. 2002). We also present the abundances of several $\mathrm{H}$ II regions: of M 33 (Kwitter \& Aller 1981; Vílchez et al. 1988), of M 31 (Blair et al. 1982), of LMC and SMC (Dufour 1984) and of the Orion 
Table 4. M 33 PNe chemical abundances in comparison with other abundances, with (N, O, S, Ar) expressed in the usual $\lg \left[\frac{X}{H}\right]+12$ units.

\begin{tabular}{|c|c|c|c|c|c|c|}
\hline & Galaxies & $\mathrm{He} / \mathrm{H}$ & $\mathrm{N} / \mathrm{H}$ & $\mathrm{O} / \mathrm{H}$ & $\mathrm{S} / \mathrm{H}$ & $\mathrm{Ar} / \mathrm{H}$ \\
\hline \multirow{7}{*}{$\mathrm{PNe}$} & $\mathrm{M} 33^{a}$ & 0.14 & 7.50 & 8.76 & 5.76 & 6.36 \\
\hline & $\mathrm{M} 31^{b}$ & 0.13 & 8.03 & 8.35 & 7.38 & 6.00 \\
\hline & Galaxy $^{c}$ & 0.115 & 8.35 & 8.68 & 6.92 & 6.39 \\
\hline & $\mathrm{LMC}^{d}$ & 0.105 & 8.07 & 8.44 & 6.51 & 5.93 \\
\hline & $\mathrm{SMC}^{d}$ & 0.107 & 7.84 & 8.24 & 6.48 & 5.62 \\
\hline & $\mathrm{Sgr}^{(e)}$ & 0.106 & 7.41 & 8.30 & 6.40 & 5.82 \\
\hline & Cen $\mathrm{A}^{f}$ & - & 8.02 & 8.39 & 7.10 & - \\
\hline \multirow{8}{*}{$\begin{array}{l}\text { H II } \\
\text { regs. }\end{array}$} & M $33^{g}$ & 0.097 & 6.98 & 8.13 & 6.81 & 5.85 \\
\hline & M $33^{h}$ & 0.083 & 7.34 & 8.55 & 6.95 & - \\
\hline & M $31^{i}$ & - & 7.63 & 8.86 & 7.11 & - \\
\hline & Orion $^{j}$ & 0.100 & 7.83 & 8.60 & 6.93 & 6.65 \\
\hline & Galaxy $^{k}$ & 0.100 & 7.57 & 8.70 & 7.06 & 6.42 \\
\hline & $\mathrm{LMC}^{k}$ & 0.085 & 6.97 & 8.43 & 6.85 & 6.20 \\
\hline & $\mathrm{SMC}^{k}$ & 0.080 & 6.46 & 8.02 & 6.49 & 5.78 \\
\hline & Solar $^{d}$ & 0.098 & 8.00 & 8.93 & 7.21 & 6.56 \\
\hline
\end{tabular}

${ }^{a}$ This work (median values of 3 PNe) ${ }^{b}$ Jacoby \& Ciardullo (1999), ${ }^{c}$ Kingsburgh \& Barlow (1994), ${ }^{d}$ Clegg (1992), ${ }^{e}$ Walsh et al. (1997), ${ }^{f}$ Walsh et al. (1999), ${ }^{g}$ Kwitter et al. (1981), ${ }^{h}$ Vílchez et al (1988), ${ }^{i}$ Blair et al. (1982), ${ }^{j}$ Rubin et al. (1991), ${ }^{k}$ Dufour (1984).

nebula (Rubin et al. 1991). Finally, the figures can be compared with the solar abundances (Clegg 1992).

The median helium abundance of our PNe is close to that of the PNe in M 31 (JC99), and slightly higher than the Milky Way PNe one. The oxygen and argon abundances are in agreement with the values obtained by KB94. They are typically lower than the solar values, as observed in Galactic PNe. The nitrogen and sulphur abundances in M $33 \mathrm{PNe}$ are unexpectedly low.

A first comment comes from the comparison of $\mathrm{PNe}$ and $\mathrm{H}$ II region chemical abundances (see Table 4). The stellar evolution theory predicts that $\mathrm{PNe}$ should have enhanced abundances of $\mathrm{He}, \mathrm{N}$ and $\mathrm{C}$ whereas the elements heavier than $\mathrm{N}$ should have abundances not appreciably different from those at the moment of the formation of their progenitor stars (cf. Iben \& Renzini 1983).

The comparison of abundances in $\mathrm{PNe}$ and $\mathrm{H}$ II regions for the galaxies of Table 4 shows that in our Galaxy, LMC and SMC the theoretical predictions in all elements are verified. The prediction appears to be verified for $\mathrm{He}$ and $\mathrm{N}$ in M 33 and for $\mathrm{N}$ in $\mathrm{M} 31$ (note that in $\mathrm{M} 31$ the $\mathrm{H}$ II region abundance of $\mathrm{He}$ is not reported) whereas for $\mathrm{O}, \mathrm{S}$, and $\mathrm{Ar}$ the numbers do not support the predictions. Since the abundances both of PNe and $\mathrm{H}$ II regions are better measured in our Galaxy and the MCs than in M 31 and M 33, we infer from the above that the abundances of $\mathrm{O}, \mathrm{S}, \mathrm{Ar}$ in $\mathrm{M} 31$ and $\mathrm{M} 33$ might not be accurate enough to claim agreement or disagreement with the theory as far as the behaviour in $\mathrm{PNe}$ versus $\mathrm{H}$ in regions is concerned. We also compare the N/O of the three PNe in M 33 to that found in $\mathrm{H}$ II regions in the same galaxy. According to Table 4, N/O is, on average, 0.05 for the three $\mathrm{PNe}$, and similar to the N/O ratios of 0.07 and 0.06 found by Kwitter et al. (1981) and Vílchez et al. (1988) for H II regions in M 33. For any other galaxy in Table 4, N/O for PNe is much larger than for $\mathrm{H}$ II regions, showing the $\mathrm{N}$ enrichment occurring in the atmospheres of intermediate mass stars. It seems that such an enrichment has not occurred for the three PNe in M 33.

In the following, we first consider the abundance of $\mathrm{S}$, which is unexpectedly low both in comparison with that in the $\mathrm{H}$ II regions of M 33 and relative to PNe in our Galaxy. Next we address the abundance of $\mathrm{N}$, which is higher than the corresponding abundance in the $\mathrm{H}$ II regions of M 33, but lower than PNe in the Galaxy.

\subsection{The sulphur abundance}

The unexpected low sulphur abundance in the PNe observed in M 33 can be explained by the fact that we are not observing the [S III] lines. In high excitation objects, such as PNe, most of the sulphur is twice ionized, and abundances derived using only [S II] lines are not representative of its total abundance, even if corrected with the nominal ICF. Thus we must consider our value as a lower limit to the true abundance of $\mathrm{S}$.

\subsection{The nitrogen abundance}

The nitrogen abundance that we found is quite low for a galaxy of normal metallicity, as M 33 is, if the abundances of the (best observed) elements like $\mathrm{He}$ and $\mathrm{O}$ are compared with the corresponding values for the Galactic PNe. Our N/H median is 7.5, which is 0.85 dex below the Galactic value, while the $\mathrm{O} / \mathrm{H}$ abundances are comparable (see Table 4). We have investigated various possible reasons of this "under-abundance". First, our chemical abundance determinations have been done using a single electron temperature $\left(T_{\mathrm{e}}[\mathrm{O} \mathrm{III}]\right)$ for all the ions. This approximation introduces errors in the calculation, especially for the abundances of low ionization ions and, in turn, of the ICFs. oxygen and argon are dominated by high ionization states, while, in the optical, nitrogen can be observed only in its $\mathrm{N}^{+}$state; thus the use of $T_{\mathrm{e}}[\mathrm{N}$ II $]=T_{\mathrm{e}}[\mathrm{O} \mathrm{III}]$ affects the determination of $\mathrm{N}$ abundances. We have tried to test this effect assuming two different electron temperatures. The low ionization temperature is usually derived from the [N II] $I(5755) / I(6583)$ ratio, which provides $T_{\mathrm{e}}[\mathrm{N}$ II]. As the [N II] $5755 \AA$ line is not observed in our spectra of M 33, we tried to estimate whether a relation between temperature from [O III] and from the $[\mathrm{N}$ II] line ratio could be established in well studied Galactic PNe. For that we have considered the extensive work of KB94. We have found that, on average, an approximately linear relation holds between the $[\mathrm{O}$ III] and [N II] temperatures. Using an error-weighted least-squares fit, we found:

$T_{\mathrm{e}}[\mathrm{N} \mathrm{III}]=0.23 T_{\mathrm{e}}[\mathrm{O} \mathrm{III}]+0.71$

where $T_{\mathrm{e}}[\mathrm{O}$ III $]$ and $T_{\mathrm{e}}[\mathrm{N}$ II $]$ are expressed in $10^{4} \mathrm{~K}$. With this relation $T_{\mathrm{e}}[\mathrm{N}$ II] can be crudely estimated for the PNe with known $T_{\mathrm{e}}[\mathrm{O}$ III]. We computed chemical abundances both with $T_{\mathrm{e}}[\mathrm{N}$ II $]=T_{\mathrm{e}}\left[\mathrm{O}\right.$ III] and with $T_{\mathrm{e}}[\mathrm{N}$ II $]$ from (1). With $T_{\mathrm{e}}[\mathrm{N} \mathrm{III}]<$ $T_{\mathrm{e}}[\mathrm{O} \mathrm{III}]$, the ionic abundance of $\mathrm{N}^{+}$is enhanced while the 
$\mathrm{ICF}(\mathrm{N})$ is lowered. The total abundance of $\mathrm{N}$, however, does not change considerably so that the appreciable nitrogen deficiency remains.

Another effect that we considered in the analysis of the possible $\mathrm{N} / \mathrm{H}$ deficiency is the accuracy of the flux measurements of the [O II] doublet at $7325 \AA$. The most critical zone of our spectra is indeed the 7200-7400 $\AA$ region, where the sky subtraction is particularly difficult. The [O II] flux is then quite uncertain. This implies possible errors on the $\mathrm{O}^{+}$abundance, in the $\operatorname{ICF}(\mathrm{N})$ and consequently on the $\mathrm{N}$ abundance. The oxygen abundance is dominated by the [O III] flux and the errors on $\mathrm{O}^{+}$ionic abundance do not affect it greatly, whereas the $\mathrm{ICF}(\mathrm{N})$ depends on the ratio between the $\mathrm{O}$ and $\mathrm{O}^{+}$abundances (see the prescriptions by KB94). We first consider the extreme case in which the sky subtraction had been plainly wrong in all three $\mathrm{PNe}$ and our mean ionic abundance of $\mathrm{O}^{+}$was only one half of the value that follows from Table 3. The $\mathrm{N}$ abundances would turn to be comparable with the mean Galactic $\mathrm{PNe} \mathrm{N} / \mathrm{H}$ value. However, if the error in the sky subtraction was not so large and systematic the possibility remains of a real under-abundance in $\mathrm{N}$ for $\mathrm{PNe}$ of $\mathrm{M} 33$. To better evaluate this possibility we have compared the behaviour of $\frac{\mathrm{N}^{+}}{\mathrm{O}^{+}}$versus $\frac{\mathrm{N}^{+}}{\mathrm{H}}$ for our PNe, which are disc PNe, with that of the Galactic sample of non-type $1 \mathrm{PNe}$ of KB94. Our PNe fall in the lowerleft part of the diagram, corresponding to the lower $\frac{\mathrm{N}^{+}}{\mathrm{O}^{+}}$values of the PNe of the KB94 sample. Our $\frac{\mathrm{N}}{\mathrm{H}}, \frac{\mathrm{N}^{+}}{\mathrm{O}^{+}}$and also $\frac{\mathrm{N}}{\mathrm{O}}$ are thus consistent with the corresponding values of the lower $\frac{\mathrm{N}^{+}}{\mathrm{O}^{+}} \mathrm{PNe}$ of the KB94 Galactic sample. If our three PNe would be representative of the M $33 \mathrm{PNe}$, their low $\frac{\mathrm{N}}{\mathrm{O}}$ would indicate a poor $\mathrm{N}$ enrichment in the atmospheres of the progenitors of the PNe, and consequently a $\mathrm{N}$ under-abundance.

Obviously, this under-abundance cannot be argued from data of only 3 PNe where the $T_{\mathrm{e}}[\mathrm{N} \mathrm{II}]$ is not directly measured, but at the same time it cannot be excluded with the present data. On the other hand, it seems there is not any evident underabundance of $\mathrm{N}$ in $\mathrm{H}$ II regions of $\mathrm{M} 33$. Their $\mathrm{N} / \mathrm{O}$ ratio is equal, within uncertainties, to that of all the galaxies in Table 4 (except the determinations by Rubin et al. 1991 for Orion). The apparently low $\frac{\mathrm{N}}{\mathrm{O}}$ ratio in the studied PNe of M 33 clearly deserves further investigation.

\section{Conclusions}

We have presented optical spectra of a sample of candidate $\mathrm{PNe}$ in the nearby spiral galaxy M 33. The location of the objects in the $\mathrm{H} \alpha /[\mathrm{S}$ II $]$ vs. $\mathrm{H} \alpha /[\mathrm{N}$ II] diagnostic diagram and their excitation parameter $R=\frac{\mathrm{R}[\mathrm{OIII}]}{\mathrm{H} \alpha+[\mathrm{NII}]}$ allowed us to confirm that 26 of the observed 36 candidate PNe with reliable spectra are very likely genuine $\mathrm{PNe}$, while seven other objects are likely compact $\mathrm{H}_{\text {II }}$ regions and another three might be SNR candidates. None of the emission objects that were classified as "uncertain" by M00 (because having a non-negligible continuum emission) seem to be PNe: three are likely $\mathrm{H}$ in regions and two SNRs. Thus, these spectra confirm the overall reliability of the criteria adopted by Magrini et al. (2000, 2001b, 2002) to select candidate PNe in galaxies of the Local Group from ground-based narrow-band imaging. The degree of contamination found for M $33 \mathrm{PNe}$ is

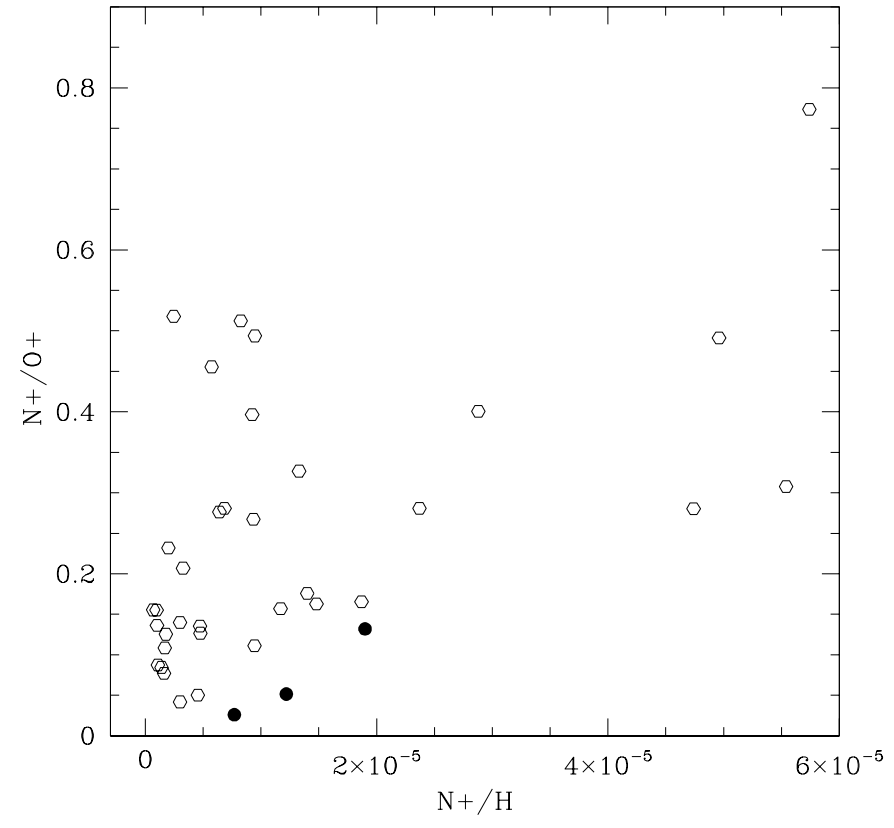

Fig. 4. $\frac{\mathrm{N}^{+}}{\mathrm{O}^{+}}$vs. $\mathrm{N}^{+} / \mathrm{H}$ : empty dots represent non-type $1 \mathrm{PNe}$ from KB94 and filled dots the $3 \mathrm{PNe}$ in $\mathrm{M} 33$.

approximately $20 \%$ and $10 \%$ for unresolved $\mathrm{H}$ II regions and SNRs, respectively.

This work also confirms the potentiality of the use of PNe for the determination of chemical abundances for the old and intermediate populations of galaxies, as an alternative to integrated light spectroscopy. With a $4 \mathrm{~m}$-class telescope one can reach the limits of the Local Group and obtain a direct abundance determination for PNe, observing the relevant emission lines for density, temperature and metallicity analysis. We present a detailed physico-chemical analysis of the three best observed $\mathrm{PNe}$ in $\mathrm{M} 33$, finding that $\mathrm{He} / \mathrm{H}, \mathrm{O} / \mathrm{H}$ and $\mathrm{Ar} / \mathrm{H}$ abundances are in agreement with the Milky Way PNe values (KB94). N/H is unexpectedly low. This might be due to the weakness of the $[\mathrm{O}$ II] lines which are strongly involved in the calculation of the correction factors needed to determine the total nitrogen abundance from single low ionization ions. On the other hand, the Galactic behaviour of $\frac{\mathrm{N}^{+}}{\mathrm{H}}$ vs. $\frac{\mathrm{N}^{+}}{\mathrm{O}^{+}}$indicates that the value of $\frac{\mathrm{N}^{+}}{\mathrm{O}^{+}}$for the PNe in M 33 is comparable with that of Galactic PNe with low nitrogen abundance. We cannot then fully exclude the possibility of a real nitrogen deficiency in PNe of M 33.

\section{References}

Acosta Pulido, J. A. 1999, in the Proc. of the "Astronomical Data Analysis and Software Systems. IX Conf.", Hawai, 1013 Oct. 1999, ed. D. Crabtree, N. Manset, \& C. Veillet

van den Bergh, S. 2000, in The Galaxies of the Local Group (Cambridge: Cambridge University Press), 269

Blair, W. P., Kirshner, R. P., \& Chevalier, R. A. 1982, ApJ, 254, 50

Brocklehurst, M. 1971, MNRAS, 153, 471

Ciardullo, R., Feldmeier, J. J., Jacoby, G. H., et al. 2002 [astro-ph/0206177]

Clegg, R. E. S. 1987, MNRAS, 229, 31 
Clegg, R. E. S. 1992, in Planetary Nebulae, ed. R. Weinberger, \& A. Acker (Dordrecht: Kluwer), IAU Symp., 155, 549

Cuisinier, F., Maciel, W. J., Köppen, J., Acker, A., \& Stenholm, B. 2000, A\&A, 353, 543

Danziger, I. J., Webster, B. L., Dopita, M. A., \& Hawarden, T. G. 1978, ApJ, 220, 458

Dopita, M. A., Vassiliadis, E., Wood, P. R., et al. 1997, ApJ, 474, 188

Dufour, R. J. 1984, in Structure and evolution of the Magellanic Clouds, ed. D. Reidel (Dordrecht: Reidel), IAU Symp., 108, 353

Ford, H., Peng, E., \& Freeman, K. 2002, in The Dynamics Structures \& History of Galaxies, ASP Conf. Ser., ed. G. S. Da Costa, \& E. M. Sadlaer

Freedman, W. L., Madore, B. F., Gibson, B. K., et al. 2001, ApJ, 553, 47

de Freitas Pacheco, J. A., Costa, R. D. D., \& Maciel, W. J. 1993, A\&A, 279, 567

Garcia Lario, P., Manchado, A., Riera, A., Mampaso, A., \& Pottasch, S. R. 1991, A\&A, 249, 223

Holmberg, E. 1958, Lund Medd. Ser., II, 136

Hummer, D. G., \& Storey, P. J. 1987, MNRAS, 224, 801

Hyung, S., Aller, L. H., Han, S., et al. 2000, JKAS, 33, 97

Iben, I. Jr., \& Renzini, A. 1983, ARA\&A, 21, 271

Jacoby, G. H., \& Ciardullo, R. 1999, ApJ, 515, 169 (JC99)

Kaler, J. B., Kwitter, K. B., Shaw, R. A., \& Browning, L. 1996, PASP, 108,980

Kingsburgh, R. L., \& Barlow, M. J. 1994, MNRAS, 271, 257 (KB94)

Kwitter, K. B., \& Aller, L. H. 1981, MNRAS, 195, 939

Magrini, L., Corradi, R. L. M., Mampaso, A., \& Perinotto, M. 2000, A\&A, 355, 713 (M00)

Magrini, L., Cardwell, A., Corradi, R. L. M., Mampaso, A., \& Perinotto, M. 2001a, A\&A, 367, 498

Magrini, L., Perinotto, M., Corradi, R. L. M., \& Mampaso, A. 2001b, A\&A, 379, 90
Magrini, L., Corradi, R. L. M., Walton, N. A., et al. 2002, A\&A, 386, 869

Mathis, J. S. 1990, ARA\&A, 28, 37

McWilliam, A. 1997, ARA\&A, 35, 503

Oke, J. B. 1974, ApJS, 27, 21

Oke, J. B. 1990, AJ, 99, 1621

Osterbrock, D. E. 1989, in Astrophysics of Gaseous Nebulae and Active Galactic Nuclei, ed. H. Knudsen, Sky, \& Telescope, 78, No. 5

Reyes, R. E. C., Steiner, J. E., \& Elizalde, F. 1997, in Planetary nebulae, Proc. of the 180th IAU Symp., ed. H. J. Habing, \& H. J. Lamers (Kluwer Academic Publishers), 471

Richer, M. G. 1993, ApJ, 415, 240

Richer, M. G., \& McCall, M. 1995, ApJ, 445, 642

Riesgo-Tirado, H., \& López, J. A. 2002, Rev. Mex. Astrofis. Astron., 12,174

Rubin, R. H., Simpson, J. P., Haas, M. R., \& Erickson, E. F. 1991, PASP, 103, 834

Sabbadin, F., \& D’Odorico, S. 1976, A\&A, 49, 119

Stanghellini, L., Shaw, R. A., Mutchler, M., Stacy, P., \& Balick, B. 2002, ApJ, in press [astro-ph/0204158]

Stasinska, G., Richer, M. G., \& Mc Call, M. L. 1998, A\&A, 336, 667

Vassiliadis, E., Dopita, M. A., Morgan, D. H., \& Bell, J. F. 1992, ApJS, 83,87

Vílchez, J. M., Pagel, B. E. J., Diaz, A. I., Terlevich, E., \& Edmunds, M. G. 1988, MNRAS, 235, 633

Walsh, J. R., Dudziak, G., Minniti, D., \& Zijlstra, A. A. 1997, ApJ, 487,651

Walsh, J. R., Walton, N. A., Jacoby, G. H., \& Peletier, R. F. 1999, A\&A, 346, 753

Zaritsky, D. 1999, in The Galactic Halo, ed. B. K. Gibson, T. S. Axelrod, \& M. E. Putman (San Francisco: Astron. Soc. Pac.), ASP Conf. Ser., 165, 34 